\title{
Teleportation with a uniformly accelerated partner
}

\author{
Paul M. Alsing* \\ High Performance Computing, Education and Research Center and \\ Center for Advanced Studies, Department of Physics and Astronomy \\ University of New Mexico, Albuquerque, NM 87131 \\ G. J. Milburn甲 \\ School of Physical Science, \\ University of Queensland, Brisbane, Australia
}

(Dated: February 1, 2008)

\begin{abstract}
In this work, we give a description of the process of teleportation between Alice in an inertial frame, and Rob who is in uniform acceleration with respect to Alice. The fidelity of the teleportation is reduced due to Unruh radiation in Rob's frame. In so far as teleportation is a measure of entanglement, our results suggest that quantum entanglement is degraded in non inertial frames.
\end{abstract}

\section{INTRODUCTION}

The large and rapidly growing field of quantum information science is a vindication of Landauer's insistence that we recognize the physical basis of information storage, processing and communication 1]. Quantum information science is based on the discovery that there are physical states of a quantum system which enable tasks that cannot be accomplished in a classical world. An important example of such a task is quantum teleportation 2]. Teleportation, like most recent ideas in quantum information science, is based squarely on the physical properties of non-relativistic quantum systems.

Recognizing that information science must be grounded in our understanding of the physical world, one is prompted to ask how relativistic considerations might impact tasks that rely on quantum entangled states. There has recently been some interest in this question for inertial frames. While Lorentz transformations cannot change the overall quantum entanglement of a bipartite state 3, 4], they can change which properties of the local systems are entangled. In particular, Gingrich and Adami [5] showed that under a Lorentz transformation the initial entanglement of just the spin degrees of freedom of two spin half particles can be transferred into an entanglement between both the spin and momentum degrees of freedom. Physically this means that detectors, which respond only to spin degrees of freedom, will see a reduction of entanglement when they are moving at large uniform velocity. Put simply, the nature of the entanglement resource depends on the inertial reference frame of the detectors. A similar result holds for photons 6 ]

In this paper however, we wish to consider quantum entanglement in non-inertial frames. In order to make the discussion physically relevant, we concentrate on a particular quantum information task; quantum teleportation. We will show that the fidelity of teleportation is

\footnotetext{
*Electronic address: alsing@hpcerc.unm.edu

†Electronic address: milburn@physics.uq.edu.au
}

compromised when the receiver is making observations in a uniformly accelerated frame. This is quite distinct from any reduction in fidelity through the Lorentz mixing of degrees of freedom noted by Gingrich and Adami [5]. Rather it is direct consequence of the existence of UnruhHawking radiation for accelerated observers. In so far as teleportation fidelity is an operational measure of quantum entanglement, our results suggest that quantum entanglement may not be preserved in non-inertial frames.

\section{UNIFORMLY ACCELERATED OBSERVERS}

\section{A. Preliminaries}

Let Alice be an inertial Minkowski observer with zero velocity, located at the point $P$ as shown in Fig.(1). Another inertial observer Bob is travelling with positive constant velocity $v<c$ in the $z$ direction with respect to Alice, and their positions are coincident at the point $P$ whereupon they each share one part of an entangled Bell state. The textbook teleportation protocol 7] proceeds as usual with Alice sending the results of her measurement to Bob at the point $Q$, say by photons, so that Bob will eventually receive them, and be able to rotate his half of the shared entangled state into the state $|\psi\rangle_{M}=\alpha|0\rangle_{M}+\beta|1\rangle_{M}$ that Alice wishes to teleport (where the $M$ subscripts denotes a Minkowski state).

The situation is drastically different for the observer Rob who travels with constant acceleration $a$ in the $z$ direction with respect to Alice. Alice's and Rob's position coincide at the point $P$ where again they instantaneously share an entangled Bell state, of which Rob takes one qubit on his journey. In Minkowski coordinates Rob's world line takes the form

$$
t_{R}(\tau)=a^{-1} \sinh a \tau, \quad z_{R}(\tau)=a^{-1} \cosh a \tau,
$$

where $\tau$ is the proper time along the world line. Rob's trajectory is a hyperbola in Minkowski space bounded by the light-like asymptotes $\mathcal{H}_{-}$and $\mathcal{H}_{+}$which represents Rob's past and future horizons with $\tau=-\infty$ and $\tau=\infty$ 


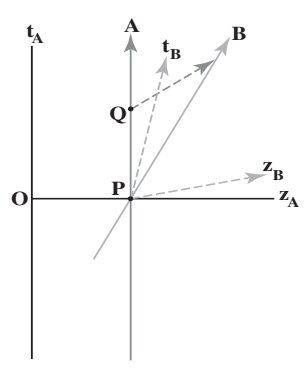

(a)

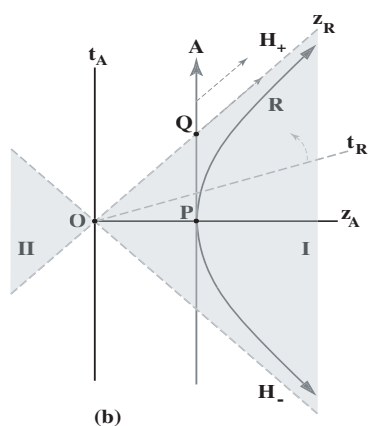

(b)
FIG. 1: (a) Minkowski diagram for the case of Alice (dark gray arrow) stationary and Bob (light gray arrow) travelling at constant velocity. Alice and Bob share an entangled Bell state at the event $P$ (see text). Alice can complete the teleportation protocol by sending classical signals to Bob at a representative event $Q$. The entanglement fidelity of a state $\Phi$ is unaltered if viewed from either Alice's or Bob's rest frame. (b) Alice (dark gray arrow) is again stationary, while Rob (dark gray hyperbola) undergoes constant acceleration. Alice and Rob share an entangled Bell state at the common point $P$. The light-like lines $\mathcal{H}_{-}$and $\mathcal{H}_{+}$form past and future particle horizon corresponding to Rob's proper times $t_{R}=-\infty$ and $t_{R}=+\infty$ respectively. At the event $Q$ Alice crosses $\mathcal{H}_{+}$ (in her finite proper time $t_{A}$ ), and can no longer communicate with Rob. Bob, however, can still send signals to Alice across $\mathcal{H}_{+}$.

respectively. The shaded region in the right half of the Minkowski plane in Fig. (1) where Rob is constrained to move is called the Right Rindler Wedge (RRW) and is labelled with the roman numeral $I$. In general, a point in the RRW can be labelled by the Rindler coordinates $(\eta, \zeta)$ which are related to Minkowski coordinates $(t, z)$ by

$$
t=\zeta \sinh \eta, \quad z=\zeta \cosh \eta
$$

where $-\infty<\eta<\infty$ and $0<\zeta<\infty$. Lines of constant $\zeta$ are hyperbolas within the RRW and lines of constant $\eta$ are straight lines through the origin. The past horizon $\mathcal{H}_{-}$corresponds to $\zeta=0, \eta=-\infty$ while the future horizon $\mathcal{H}_{+}$corresponds to $\zeta=0, \tau=\infty$.

With the same coordinate transformation given in Eq.(2), the region $-\infty<\zeta<0$ and $-\infty<\eta<\infty$ is called the Left Rindler Wedge (LRW) and is labeled by the roman numeral $I I$. In this region, the lines of constant $\eta$ run in the opposite sense than in $I$. Region $I$ is causally disconnected from $I I$ and no signal from one region can propagate into the other region. The metric for Minkowski space is given by

$$
d s^{2}=d z^{2}-d t^{2}=d \zeta^{2}-\zeta^{2} d \eta^{2} .
$$

It is well appreciated now $[8,9,10,11,12,13$ that the quantization of fields in Minkowski and Rindler coordinates are inequivalent, implying that the RRW vacuum seen by Rob $|0\rangle_{I}$ is different than the Minkowski vacuum seen by Alice $|0\rangle_{M}$. The celebrated result of Davies and Unruh 9 ] is that the Minkowski vacuum can be written in terms of the region $I$ and $I I$ states (for a scalar field) as

$$
|0\rangle_{M}=\prod_{\Omega, \vec{k}_{\perp}}\left(1-e^{-2 \pi \Omega}\right)^{-1 / 2} \sum_{n=0}^{\infty} e^{-2 \pi \Omega n}\left|n_{\Omega, \vec{k}_{\perp}}\right\rangle_{I} \otimes\left|n_{\Omega,-\vec{k}_{\perp}}\right\rangle_{I I},
$$

where $\Omega \equiv \omega_{R} /(a / c)$ with $\omega_{R}$ the frequency of a Rindler particle. The Minkowski vacuum as given by Eq. (4) is a two-mode squeezed state [14 which for each mode $\left(\Omega, \vec{k}_{\perp}\right)$ has the general form

$$
|0\rangle_{M} \sim \frac{1}{\cosh r} \sum_{n=0}^{\infty} \tanh ^{n} r|n\rangle_{I} \otimes|n\rangle_{I I}
$$

with

$\cosh r=\left(1-e^{-2 \pi \Omega}\right)^{-1 / 2}, \sinh r=e^{-\pi \Omega}\left(1-e^{-2 \pi \Omega}\right)^{-1 / 2}$.

Note that $|0\rangle_{M}$ can be written as $S(r)|0\rangle_{I} \otimes|0\rangle_{I I}$ where the two-mode squeezing operator is given by $S(r) \equiv$ $\exp \left[r\left(b_{I} b_{I I}-b_{I}^{\dagger} b_{I I}^{\dagger}\right)\right]$ 14]. The evolution of a Minkowski state vector is affected by the unitary operator $e^{-i H_{M} t}$ where for a single mode (ignoring transverse momentum degrees of freedom) $H_{M}=\hbar \omega_{M} a_{M}^{\dagger} a_{M}$. For Rindler states the evolution proceeds via $e^{-i H_{R} \tau}$ where

$$
\begin{aligned}
H_{R} & \equiv H_{I}-H_{I I} \\
H_{I} & =\hbar \omega_{R} b_{I}^{\dagger} b_{I}, \quad H_{I I}=\hbar \omega_{R} b_{I I}^{\dagger} b_{I I} .
\end{aligned}
$$

The minus sign in Eq.(8) stems from the sense of time essentially flowing "backwards" in region $I I$ (i.e. for $a<$ $0, \eta(\tau)=a \tau$ is a decreasing function of $\tau$ ).

For Rob, who lives in region $I$, all his observables can be written solely in terms of $b_{I}$ and $b_{I}^{\dagger}$ operators. Since he is causally disconnected from region $I I$, Rob must reduce any density matrix describing both Rindler wedges to one appropriate to region $I$ only, by tracing out over region $I I$. Thus he perceives the Minkowksi vacuum as a thermal state,

$$
\rho_{|0\rangle_{M}}^{(I)} \equiv \operatorname{Tr}\left(|0\rangle_{M}\langle 0|\right)=\left(1-e^{-2 \pi \Omega}\right) \sum_{n=0}^{\infty} e^{-2 \pi \Omega n}|n\rangle_{I}\langle n| .
$$

The exponential terms can be written as $\exp \left(-\hbar \omega_{R} / k_{B} T_{U}\right)$ with the Unruh temperature $T_{U}$ is given by (in units of $k_{B}=1$ )

$$
T_{U} \equiv \frac{\hbar a}{2 \pi c}=\frac{\hbar}{2 \pi c \zeta_{0}}
$$

where $\zeta(\tau)=\zeta_{0}=1 / a$ is the constant Rindler position coordinate of Rob's stationary world line. 


\section{B. Relationship between Minkowski and Rindler modes}

We will use two-photon states of the electromagnetic field [15] for the Bell state and so must consider Fock states, other than the vacuum state, for the Rindler observer. This is easily done by a consideration of how the creation and anihilation operators transform. The relationship between the Minkowski and Rindler modes is given by the Bogoliubov transformation

$$
b_{\Omega, \vec{k}_{\perp}}^{(\sigma)}=\int d^{3} k^{\prime}\left(\alpha \alpha_{k k^{\prime}}^{(\sigma)} a_{k^{\prime}}+\beta_{k k^{\prime}}^{(\sigma)} a_{k^{\prime}}^{\dagger}\right)
$$

where the notation of 11] has been adopted, namely $\sigma=$ $(+,-)$ refers to region $I$ and $I I$ respectively, $k=\left(\Omega, \vec{k}_{\perp}\right)$ and $k^{\prime}=\left(\vec{k}_{\perp}, k^{3}\right)$. Modes in Minkowski space are specified by the wave vector $\vec{k} \equiv\left(\vec{k}_{\perp}, k^{3}\right)$ where $\vec{k}_{\perp}=\left(k^{1}, k^{2}\right)$ are the components of the momentum perpendicular to the direction of Rob's acceleration. The Minkowski frequency is given by $\omega_{\vec{k}}=\sqrt{m^{2}+\vec{k}^{2}}$. Modes in Rindler space are specified by a positive energy Rindler frequency $\Omega$ and $\vec{k}_{\perp}$. The Bogoliubov transformation can be put into a more transparent form by introducing the $U n$ ruh modes $d_{\Omega, \vec{k}_{\perp}}^{(\sigma)}$ and $d_{\Omega, \vec{k}_{\perp}}^{(\sigma) \dagger}$. The Unruh modes arise by considering the fourier transform of the usual Minkowski plane waves $\left[(2 \pi)^{3} 2 \omega_{\vec{k}}\right]^{-1 / 2} \exp \left(\vec{k} \cdot \vec{x}-\omega_{\vec{k}} t\right)$ in terms of the Rindler proper time $\tau$ [13]. These complete, orthonormal set of modes exits over all of Minkowski space and can be "patched together" to form the two complete orthonormal set of Rindler modes which have finite support in either region $I$ or region $I I$. The physical significance of the Unruh modes is that they diagonalize the generator of Lorentz boosts [1], which in Minkowski coordinates is given by

$$
M^{\alpha \beta}=\int d^{3} x\left(x^{\alpha} T^{0 \beta}-x^{\beta} T^{0 \alpha}\right) .
$$

The restriction of the generator of boosts in the $z$ direction to region $I$ gives the Rindler Hamiltonian $H_{R}=$ $\left.M^{03}\right|_{I}$

The relationship between the Unruh modes and the Minkowski modes is given by [1]

$$
a_{\vec{k}_{\perp}, k^{3}}=\sum_{\sigma} \int_{0}^{\infty} d \Omega p_{\Omega}^{(\sigma) *}\left(k^{3}\right) d_{\Omega, \vec{k}_{\perp}}^{(\sigma)}
$$

which can be inverted to give

$$
d_{\Omega, \vec{k}_{\perp}}^{(\sigma)}=\int_{-\infty}^{\infty} d k^{3} p_{\Omega}^{(\sigma)}\left(k^{3}\right) a_{\vec{k}_{\perp}, k^{3}} .
$$

In the above expression, the functions $p_{\Omega}^{(\sigma)}\left(k^{3}\right)$ form a complete orthonormal set and are given by

$$
p_{\Omega}^{(\sigma)}\left(k^{3}\right)=\frac{1}{\left(2 \pi \omega_{\vec{k}}\right)^{1 / 2}}\left(\frac{\omega_{\vec{k}}+k^{3}}{\omega_{\vec{k}}-k^{3}}\right)^{i \sigma \Omega / 2}
$$

which are essentially phase factors. Since by Eq. (13) the Unruh annihilation operator is a sum over only Minkowski annihilation operators, it too annihilates the Minkowski vacuum.

$$
a_{\vec{k}_{\perp}, k^{3}}|0\rangle_{M}=0, \quad d_{\Omega, \pm \vec{k}_{\perp}}^{(\sigma)}|0\rangle_{M}=0 .
$$

Finally, the Unruh modes are related in a natural way to the Rindler modes through the following Bogoliubov transformation

$$
\left[\begin{array}{c}
d_{\Omega, \vec{k}_{\perp}}^{(+)} \\
d_{\Omega,-\vec{k}_{\perp}}^{(-) \dagger}
\end{array}\right]=\left[\begin{array}{cc}
\cosh r & -\sinh r \\
-\sinh r & \cosh r
\end{array}\right]\left[\begin{array}{c}
b_{\Omega, \vec{k}_{\perp}}^{(+)} \\
b_{\Omega,-\vec{k}_{\perp}}^{(-)}
\end{array}\right]
$$

with the hyperbolic functions of $r$ related to the Rindler frequency $\Omega$ by Eq. (6). The operators $b^{(+)}$and $b^{(-)}$annihilate the RRW vacuum $|0\rangle_{+}$and LRW vacuum $|0\rangle_{-}$ respectively, and commute with each other.

By Eq. (12) we see that a given Minkowski mode of frequency $\omega_{\vec{k}}$ is spread over all positive Rindler frequencies $\Omega$, as a result of the Fourier transform relationship between $a_{\vec{k}_{\perp}, k^{3}}$ and $d_{\Omega, \vec{k}_{\perp}}^{(\sigma)}$. We now simplify our analysis by considering the effect of teleportation of the state $|\psi\rangle_{M}=\alpha|0\rangle_{M}+\beta|1\rangle_{M}$ by the Minkowski observer Alice to a single Rindler mode of the RRW observer Rob. That is, we consider only the mode $\left(\Omega, \vec{k}_{\perp}\right)$ in region $I$ which is distinct from the mode $\left(\Omega,-\vec{k}_{\perp}\right)$ in the same region. As such, we can consider only the $\sigma=(+)$ contribution and drop the unessential phase factors in Eq.(12). The single Rindler mode component of the Minkowski vacuum state we are interested is then

$$
|0\rangle_{M} \rightarrow \frac{1}{\cosh r} \sum_{n=0}^{\infty} \tanh ^{n} r\left|n_{\Omega, \vec{k}_{\perp}}\right\rangle_{I} \otimes\left|n_{\Omega,-\vec{k}_{\perp}}\right\rangle_{I I} .
$$

The relevant Bogoliubov transformation can now be written as

$$
a_{\vec{k}_{\perp}, k^{3}}^{\dagger} \rightarrow d_{\Omega, \vec{k}_{\perp}}^{(+) \dagger}=\cosh r b_{\Omega, \vec{k}_{\perp}}^{(+) \dagger}-\sinh r b_{\Omega,-\vec{k}_{\perp}}^{(-)} .
$$

From here on we drop all the frequency and momentum subscripts and replace the labels \pm by $I$ and $I I$, keeping in mind the full definitions in Eq.(17) and Eq.(18).

\section{TELEPORTATION FROM A MINKOWSKI OBSERVER TO A RINDLER OBSERVER}

Let us first begin by briefly recalling the usual teleportation protocol, between Minkowski observers Alice and Bob [Fig.(17)], as given in [7]. Our two qubit entangled state will be encoded as entangled Fock states of the electromagnetic field. Alice wishes to teleport the state $|\psi\rangle_{M}=\alpha|0\rangle_{M}+\beta|1\rangle_{M}$ to Bob. Let Alice and Bob share the entangled Bell state $\left|\beta_{00}\right\rangle_{M}=$ $1 / \sqrt{2}\left(|0\rangle_{M} \otimes|0\rangle_{M}+|1\rangle_{M} \otimes|1\rangle_{M}\right)$. The input state to the system is then $\left|\Psi_{0}\right\rangle_{M}=|\psi\rangle_{M}\left|\beta_{00}\right\rangle_{M}$. Alice 
performs a CNOT gate on $|\psi\rangle_{M}$ and her portion of $\left|\beta_{00}\right\rangle_{M}$, and then passes the first qubit of the output state through a Hadammard gate. Upon making a joint projective measurement on her two qubits with the result $|l\rangle_{M} \otimes|m\rangle_{M}$ with $l, m=\{0,1\}$, the full state is projected into $|l\rangle_{M} \otimes|m\rangle_{M} \otimes\left|\phi_{l, m}\right\rangle_{M}$ where Bob's state is given by $\left|\phi_{l m}\right\rangle_{M} \equiv x_{l m}|0\rangle_{M}+y_{l m}|1\rangle_{M}$. Here we have defined the four possible outcomes as $\left(x_{00}, y_{00}\right)=(\alpha, \beta)$, $\left(x_{01}, y_{01}\right)=(\beta, \alpha),\left(x_{10}, y_{10}\right)=(\alpha,-\beta)$, and $\left(x_{11}, y_{11}\right)=$ $(-\beta, \alpha)$. After receiving the classical information $\{l, m\}$ of the result of Alice's measurement, Bob can rotate his qubit of the entangled state into $|\psi\rangle_{M}$ by applying the operations $Z_{M}^{l} X_{M}^{m}$ to $\left|\phi_{l m}\right\rangle_{M}$. The fidelity of the teleported state is unity in this idealized situation.

Alice now wishes to perform this same teleportation protocol with the uniformly accelerated Rob. The calculation proceeds straightforwardly once we remember two things. First, the states $|0\rangle_{M}$ and $|1\rangle_{M}=a_{M}^{\dagger}|0\rangle_{M}$ of the initial state $\left|\Psi_{0}\right\rangle_{M}$ associated with Rob must be expanded in terms of the Rindler states $|n\rangle_{I} \otimes|m\rangle_{I I}$. This can be accomplished by using the single mode Minkowski vacuum as given in Eq. (17) and the Bogoliubov transformation Eq. (18). Here we are imagining the situation where Rob has instantaneously accelerated to the value $a$ at $\tau=0$ at the point $P$ in Fig.(10) where Alice and Rob initially share the entangle state $\left|\beta_{00}\right\rangle_{M}$. Second, since the result of Rob's acceleration inextricably creates a particle horizon which keeps him causally disconnected from region $I I$, his final state is produced by tracing out over region $I I$. Thus, since any Minkowski Fock state is a correlated state of region $I$ and $I I$ Fock states, there is no hope of completely teleporting the state $|\psi\rangle_{M}=\alpha|0\rangle_{M}+\beta|1\rangle_{M}$ in the presence of this partial trace operation by Rob. However, the best state we might expect Rob to obtain as an end product of the teleportation protocol would be the region $I$ analogous version of the Minkowski transported state $|\psi\rangle_{M}$, namely

$$
|\psi\rangle_{I}=\alpha|0\rangle_{I}+\beta|1\rangle_{I}
$$

We might call this a thermally teleported state since Rob perceives all Minkowski states through the haze of the thermal vacuum that he moves through. If $\rho_{l m}^{(I)}$ is Rob's density matrix after Alice performs the operations on her qubits, then we can measure the fidelity of the thermally teleported state as

$$
F^{(I)} \equiv \operatorname{Tr}_{I}\left(|\psi\rangle_{I}\langle\psi| \rho^{(I)}\right)={ }_{I}\left\langle\psi\left|\rho^{(I)}\right| \psi\right\rangle_{I} .
$$

Using Eq. (5) for $|0\rangle_{M}$ and Eq. (18) for $a_{M}^{\dagger}$ we find

$$
|1\rangle_{M}=\frac{1}{\cosh ^{2} r} \sum_{n=0}^{\infty} \tanh ^{n} r \sqrt{n+1}|n+1\rangle_{I} \otimes|n\rangle_{I I}
$$

When Alice sends the result of her measurement $\{l, m\}$, which can be received by Rob, if Alice has not yet crossed Rob's future horizon $\mathcal{H}_{+}$, Rob's state will be projected into

$$
\begin{aligned}
\rho_{l m}^{(I)} & \equiv \sum_{n=0}^{\infty}{ }_{I I}\left\langle n \mid \phi_{l m}\right\rangle_{M}\left\langle\phi_{l m} \mid n\right\rangle_{I I} \\
& =\sum_{n=0}^{\infty} \frac{\tanh ^{2 n} r}{\cosh ^{2} r}\left[\left(\left|x_{l m}\right|^{2}+n \frac{\left|y_{l m}\right|^{2}}{\sinh ^{2} r}\right)|n\rangle_{I}\langle n|+\right. \\
& +\frac{x_{l m} y_{l m}^{*}}{\cosh r} \sqrt{n+1}|n+1\rangle_{I}\langle n| \\
& \left.+\frac{x_{l m}^{*} y_{l m}}{\cosh r} \sqrt{n+1}|n\rangle_{I}\langle n+1|\right] .
\end{aligned}
$$

Note that as the acceleration becomes large i.e. $r \rightarrow$ $\infty$, Rob's state is driven into the thermal vacuum state $\rho_{|0\rangle_{M}}^{(I)}$ of Eq.(9) and all information has been lost due to thermalization.

Let us now compute the fidelity in Eq.(20) with the state $\left|\phi_{l m}\right\rangle_{I}=x_{l m}|0\rangle_{I}+y_{l m}|1\rangle_{I}$ with $\left|x_{l m}\right|^{2}+\left|y_{l m}\right|^{2}=1$, which is the penultimate state before Rob would perform the appropriate rotation to attempt to transform his half of the (accelerated) entangled Bell state to its final form $|\psi\rangle_{I}$. We obtain

$$
\begin{aligned}
F_{l m}^{(I)} & ={ }_{I}\left\langle\phi_{l m}\left|\rho_{l m}^{(I)}\right| \phi_{l m}\right\rangle_{I} \\
& =\frac{1}{\cosh ^{2} r}\left[\left|x_{l m}\right|^{4}+\left(\tanh ^{2} r\left|x_{l m}\right|^{2}+\frac{\left|y_{l m}\right|^{2}}{\cosh ^{2} r}\right)\left|y_{l m}\right|^{2}\right. \\
& \left.+2 \frac{\left|x_{l m}\right|^{2}\left|y_{l m}\right|^{2}}{\cosh r}\right],
\end{aligned}
$$

which is essentially a projection onto the $\left\{|0\rangle_{I},|1\rangle_{I}\right\}$ subspace of $\rho_{l m}^{(I)}$.

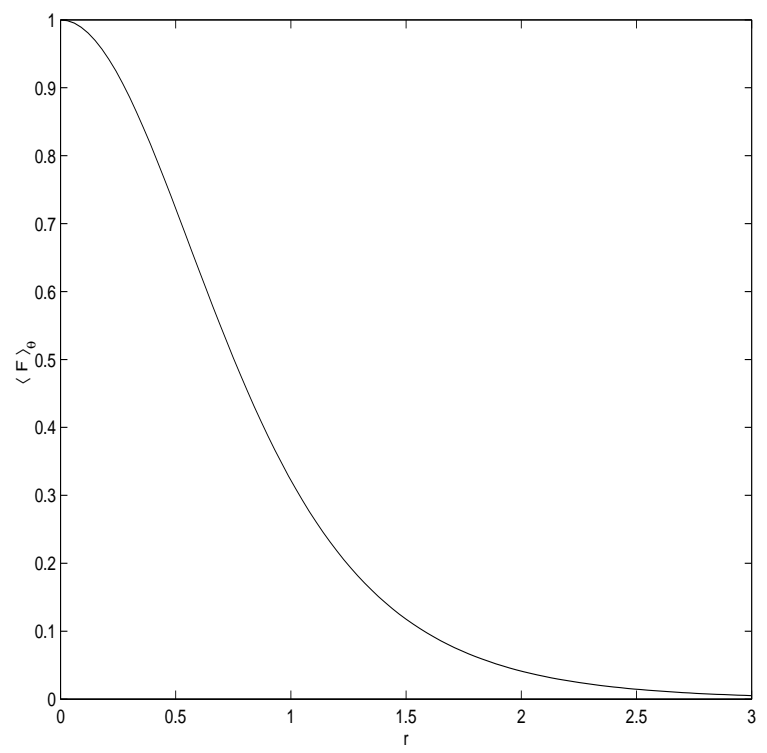

FIG. 2: Averaged Fidelity $1 / \pi \int_{0}^{\pi} d \theta F_{l m}^{(I)}(r, \theta)$ over all possible input states for $x_{l m}=\cos \theta, y_{l m}=\sin \theta$. Note that $r$ scales directly with the acceleration $a$, so that $r=0$ corresponds to $a=0$. 
In Fig.(2) we plot $F_{l m}^{(I)}$ averaged over all possible input states using the parameterization $x_{l m}=\cos \theta$ and $y_{l m}=$ $\sin \theta$. At $r=0$, corresponding to $a=0$, we are back to the case of teleportation between Alice and Bob in Minkowski space, and the fidelity is unity. Using the definition of $\Omega$ and $r$ in Eq.(6) we find

$$
\begin{aligned}
\tanh r & =\exp \left[-\pi \omega_{R} /(a / c)\right] \quad \text { or } \\
r & \approx \exp \left[-\pi \omega_{R} /(a / c)\right] \quad \text { for } r \rightarrow 0 .
\end{aligned}
$$

For terrestrial experiments $a=g \sim 10 \mathrm{~m} / \mathrm{s}^{2}$ and $a / c \sim$ $10^{-8} s^{-1}$ is such a small frequency, that for all frequencies $\omega_{R}$ of physical interest the fidelity is near unity with incredible precision, (for $r=10^{-3}$ the fidelity is is unity to within one part in $10^{6}$, and this still corresponds to unphysically large accelerations). Near the event horizon of a black hole appreciable accelerations can be obtained such that the reduction of the fidelity from unity could be observed. In that case an analogous teleportation scheme could be defined with Rob, stationary, outside the event horizon and Alice freely falling into the hole.

In the above we have ignored the time evolution of the states, namely that Alice's qubits evolve according to $e^{-i H_{M} t}$ while Rob's qubits evolve via $e^{-i H_{R} \tau}$, as discussed in the previous section. A simple calculation reveals that only change from the above analysis is that $y_{l m} \rightarrow y_{l m} e^{-i \Omega \tau}$ in Eq. (21). However, since the fidelity in Eq. (23) depends only upon $\left|y_{l m}\right|^{2}$, this phase factor does not contribute to the final result. From Rob's point of view, he is able to receive the result of Alice's measurement for all of his eternity, i.e $0<\tau<\infty$. From Alice's point of view, she cross Rob's future horizon $\mathcal{H}_{+}$ in a finite time $t=c / a$, after which even light signals will not reach Rob (see point $Q$ in Fig.(1))). For her, the teleportation protocol stops since she can no longer communicate the results of her measurements to Rob. However, for all of her eternity, i.e. $0<t<\infty$ she can still receive persistent requests from Rob to send the information. $\mathcal{H}_{+}$is analogous to the "one-way membrane" of an black hole event horizon, which Rob has "fallen" through.

It is of some interest to consider the reduction of fidelity in terms of entropy. In Fig.(3) we plot the von Neumann entropy $S=-\operatorname{Tr}(\rho \log \rho)$ of Rob's premeasurement state, post-measurement state upon learning the result of Alice's measurement, and the vacuum state, as a function of $r$ (measured in bits). The premeasurement state is obtained from Eq.(22) by summing $(l, m)$ over all four possible input states, which reduces it to a diagonal density matrix. The post-measurement state is given by Eq.(22) with the input state to the teleportation protocol chosen to be $|\psi\rangle_{M}=1 / \sqrt{2}\left(|0\rangle_{M}+\right.$ $\left.|1\rangle_{M}\right)$.

For $r=0$, corresponding to $a=0$, Rob's premeasurement state is just $\frac{1}{2} \mathbf{I}_{2 \times 2}$, half the 2 -by- 2 unit matrix in the $\left\{|0\rangle_{M},|1\rangle_{M}\right\}$ sector (since $|n\rangle_{I} \equiv|n\rangle_{M}$ in this limit) and zero elsewhere, while the vacuum is just the normal Minkowski vacuum. Note that $S_{\text {pre }}(0)=1$ expressing Rob's (or here Bob's at $r=0$ ) complete ignorance of the outcome of Alices's measurement. As $r$ increases all entropies increase since the thermalization eventually drives all states to the vacuum (in the limit $r \rightarrow \infty$ ) with equally weighted states, where all information is lost. The energy for this thermalization, of course, comes from the work supplied by the agent causing Rob's acceleration.

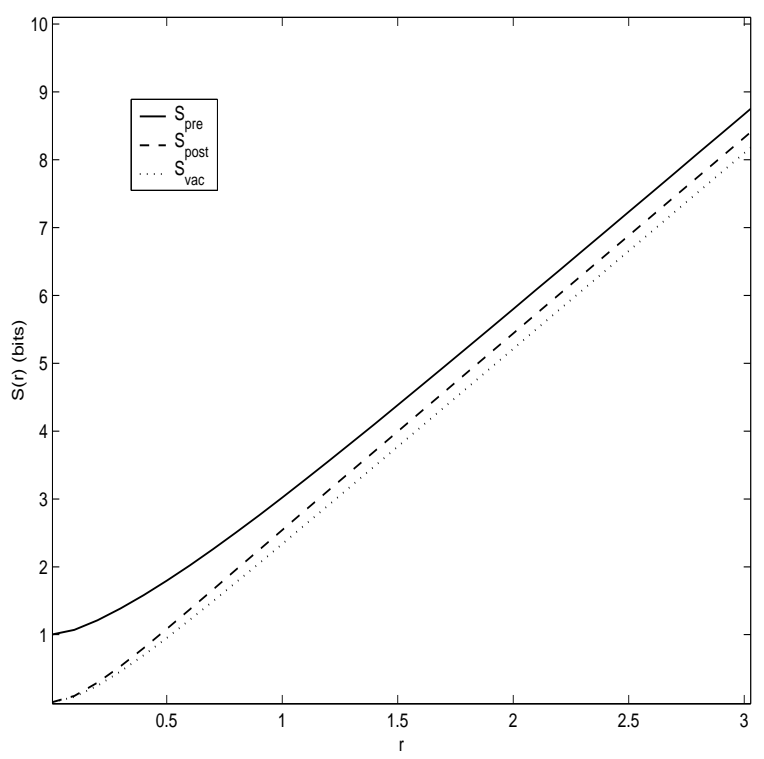

FIG. 3: Von Neumann entropies of Rob's pre-measurement state $S_{\text {pre }}$ (solid), post-measurement state $S_{\text {post }}$ (dashed), and the vacuum $S_{\text {vac }}$ (dotted).

Also plotted in Fig.(3) is the entropy for Rob's postmeasurement state $S_{\text {post }}$. At $r=0$ Rob (or Bob) would absolutely know the state that was teleported to him and thus gain 1 bit of information (the distance between the solid and dashed curve). However, as the acceleration increases, this information gain decreases from unity to zero as the Unruh temperature increases. This is seen as the solid curve in Fig.(4) which plots $\Delta S_{\text {gain }} \equiv S_{\text {pre }}-$ $S_{\text {post }}$.

Though $\Delta S_{\text {gain }} \equiv S_{\text {pre }}-S_{\text {post }}$ appears to be levelling out for large $r$ in Fig.(4), it is doing so only very slowly. Since, as mentioned above, all states are driven to the infinite temperature vacuum as $r \rightarrow \infty$, these curves must eventually merge. It is curious that they do so slowly. The dashed curve in Fig.(4) is a 2-state model which uses only the (normalized) $\left\{|0\rangle_{I},|1\rangle_{I}\right\}$ sector of Rob's post-measurement state. The entropies for this two-state model and the vacuum can then be computed analytically and the resulting entropy difference $\Delta S_{\text {gain }}^{T S M}$ agrees well with the numerical calculation $\Delta S_{\text {gain }}$ for small values of $r \leq 1 / 2$. This two state model thermalizes much faster than the full post-measurement state and so approaches zero more rapidly due the finite number of states (2) used. The interesting point to note is that even after Rob receives Alice's classical information about the result of her 
measurement Rob is prevented from regaining the full 1 bit of information if his acceleration is non zero. This is another way to see that from Rob's perspective information appears to be lost.

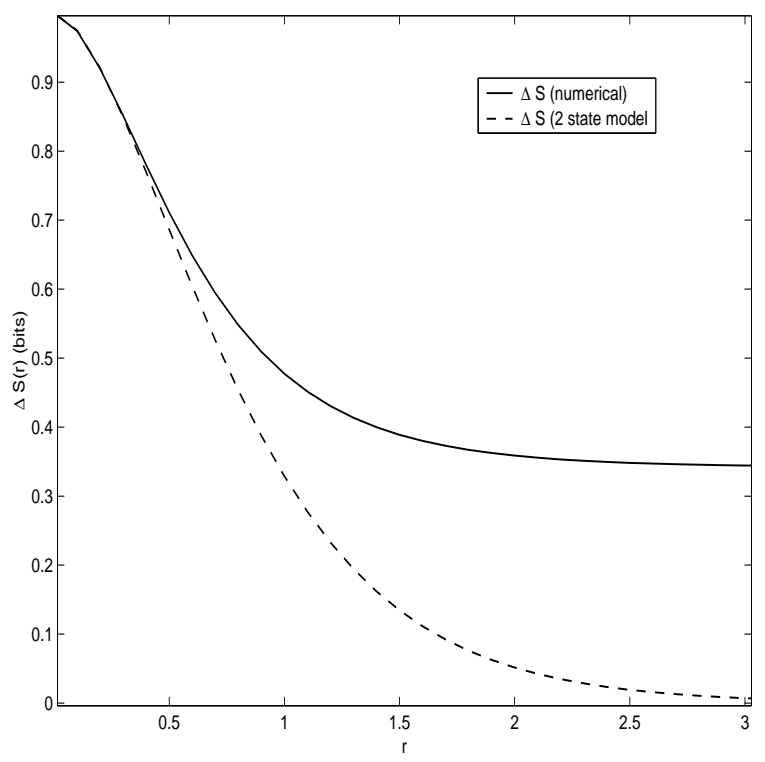

FIG. 4: Rob's entropy information gain (in bits) $\Delta S_{\text {gain }}=$ $S_{\text {pre }}-S_{\text {post }}$ upon receiving the Alice's measurement results: numerical (solid) and $\Delta S_{\text {gain }}^{T S M}$ (dashed) for a 2-state model using the $\left\{|0\rangle_{I},|1\rangle_{I}\right\}$ sector from Rob's post measurement state, with $x_{l m}=y_{l m}=1 / \sqrt{2}$.

\section{DISCUSSION AND CONCLUSIONS}

The main issues of teleportation between an inertial Minkowksi observer Alice and a non-inertial, uniformly accelerated Rindler observer Rob are two fold. First, as a result of the acceleration, the Minkowski vacuum that Rob moves through (for a single Rindler mode $\left(\Omega, \vec{k}_{\perp}\right)$ ) can be written as a two-mode squeezed state with the component Fock states existing in causally separated regions $I$ and $I I$. Second, as a result of this fact, Rob's perceives the Minkowski vacuum as a pure thermal state of temperature $T_{U}$ as the inevitable result of his complete ignorance of region $I I$.

In an attempt to teleport a state $|\psi\rangle_{M}=\alpha|0\rangle_{M}+$ $\beta|1\rangle_{M}$ to Rob, the best we can expect Rob to recover at the end of the protocol is $|\psi\rangle_{I}=\alpha|0\rangle_{I}+\beta|1\rangle_{I}$. In this work we have calculated the fidelity of the state Rob receives with this best possible result $|\psi\rangle_{I}$. We have demonstrated how the fidelity decreases with increasing acceleration until at high temperatures all information is lost and Rob perceives only the thermalized vacuum state.

The model investigated here is equivalent to teleportation through two channels, one of which is free space for Alice and the second which involves parametric down conversion with the following caveat described below. In the second channel, a signal mode $I$ and an idler mode $I I$ experience a squeezing Bogoliubov transformation analogous to Eq.(16) [16]. Here $r$ is proportional to the coupling strength between the signal and idler mode times the length of the crystal through which the parametric down conversion takes place; higher interaction strengths and/or longer interaction lengths corresponds to a higher Unruh temperature. The caveat is that Rob, acting as say the signal mode, has no access to information about the idler and therefore must trace out this information. Performing the teleportation protocol in such a system is exactly analogous to teleportation between a Minkowski and Rindler observer as considered in this work. In the parametric down conversion model, Rob can choose to ignore the idler information thus mimicking a Rindler observer. However, for an accelerated observer, the existence of the horizons $\mathcal{H}_{ \pm}$is of fundamental importance. Since region $I$ and $I I$ are causally disconnected, there is no way, even in principle, for Rob to have any information about region $I I$, and thus his state is always a reduced density matrix appropriate for region $I$.

We have given an explanation of the reduction of teleportation fidelity in terms of the Unruh radiation seen by Rob in his frame. Note that this is an operationally meaningful statement as Rob can attempt to verify that he has not received the desired state $\left(x_{l m}|0\rangle_{I}+y_{l m}|1\rangle_{I}\right)$ by local verification measurements (e.g. a single photon interference experiment), and then send the results to Alice. It would be quite easy to arrange a situation whereby Alice could tell unambiguously that Rob had received the wrong state. From an operational point of view Alice would conclude that the shared entangled resource has become decohered. It is well know that entanglement is a fragile resource in the presence of environmental decoherence. It appears also to be a fragile resource when one of the entangled parties undergoes acceleration. While the degree of decoherence is exceedingly small for practical accelerations, the apparent connection between space time geometry and quantum entanglement is intriguing.

Added Note During the preparation of this work, the authors became aware of the recent paper by Anderson et al 17] which also discusses teleportation and the Unruh vacuum. However, that work considers a physically different situation than the one presented here. The authors use the mirror modes of Audretsch and Müller [18] and consequently have the accelerated observers travelling on oppositely directed hyperbolas, with Alice in region $I$ and Bob in the causally disconnected region $I I$. The teleportation protocol is then interpreted from the point of view of a Minkowski observer Mork. In this work, we consider a setup between observers, one stationary, the other accelerated, who remain causally connected to each other during the teleportation protocol. 


\section{Acknowledgments}

The authors wish to thank Jon P. Dowling for stimu- lating discussions on this and other topics in relativistic quantum information theory.
[1] R. Landauer, Physics Today, 44:23-29, May (1991).

[2] C.H. Bennett, G. Brassard, C. Crépeau, R. Jozsa, A. Peres, W.K. Wooters, Phys. Rev. Letts. 70, 1895 (1993).

[3] A. Peres,P.F. Scudo and D.R. Terno, Phys. Rev. Lett., 88, 230402 (2002); A. Peres and D.R. Terno quant-pn/0208128 (2002).

[4] P.M. Alsing and G.J. Milburn, Quantum Inf. Comput. 2, 487 (2002).

[5] R.M.Gingrich and C. Adami, Phys. Rev. Lett. 89, 270042 (2002).

[6] R.M. Gingrich, A. J. Bergou and C. Adami quant-ph/0302095 (2003).

[7] M.A. Nielsen and I. Chuang, Quantum Computation and Quantum Information, Cambridge Univ. Press, p25-28, (2000).

[8] S.A. Fulling, Phys. Rev. D7, 2850 (1973).

[9] P.C.W. Davies, J. of Phys. A8, 609 (1975); W.G. Unruh, Phys. Rev. D14, 870 (1976);

[10] N.D. Birell and P.C.W Davies, Quantum fields in curved space, Cambridge Univ. Press (1982).

[11] S. Takagi, Progress of Theoretical Physics Supplement No. 88, 1-142 (1986).

[12] G. 'T Hooft, Acta Physica Polonica B19, 187 (1988).

[13] L.N. Pringle, Phys. Rev D39, 2178 (1989).

[14] D.F. Walls and G.J. Milburn, Quantum Optics, SpringerVerlag, New York (1994).

[15] The quantization of field modes discussed this work has actually been carried out for scalar particles. However, the main results of this work are not modified by the inclusion of spin or helicity degrees of freedom.

[16] B. Yurke, Squeezed Light, unpublished lectures notes, Institute of Optics, University of Rochester, Rochester, NY, (1989).

[17] X. Anderson, S.J. van Enk and T. Rudolph, quant-ph/0302091.

[18] J. Audretsch and R. Müller, Phys. Rev. D 49, 4056 (1994). 